\title{
Comparison of Coordinate Data by Radio-Technical Systems for Sustainable Development of Territories
}

\author{
S. V. Bozhko*, E. V. Konovaltsev, N. A. Kupriyanov, S. V. Stadnik \\ Krasnodar Higher Military Aviation School of Pilots named after Hero of the Soviet Union A. K. Serov, Krasnodar, \\ Russian Federation \\ *Corresponding author. Email: bsvinfo60@mail.ru
}

\begin{abstract}
Some aspects of coordinate data comparison by radio-technical systems are considered. The features of the initial data preparation are described and the main calculated ratios are provided. Possible implementation of comparison of coordinate data by spatially separated radio-technical systems to improve the quality of location, navigation, remote sensing, television and communications is shown.
\end{abstract}

Keywords: measurement error, total electron content, a priori coordinate information.

\section{INTRODUCTION}

It is known that sustainable development of territories involves limiting the negative impact of economic and other human activities on the environment, as well as ensuring protection and rational use of natural resources in the interests of current and future generations [1]. However, increase in the number of objects [2] caused by human activity in near-Earth airspace (NEAS) requires an increase in the number of various ground-based observation systems [3, 4]. At present, the largest contribution to the scope of coordinate data obtained from NEAS falls on radar observation systems (ROS) [5]. At the same time, functioning of the ROS, regardless of its purpose and the tasks being solved, indirectly affects the environment and requires a significant amount of electricity [6], thereby going against the concept of sustainable development of territories.

In addition, the accuracy of determining the coordinates by radar methods is influenced by a number of factors, considering of which, when processing the measurement results, makes it possible to achieve the specified measurement accuracy. Experience of operating various radio-technical systems has shown that sporadic ionization of near-Earth space has the most significant effect on reducing the measurement accuracy $[7,8]$. This is explained by the application in the ROS of approaches based on long-term prediction of the parameters of the radio wave propagation medium, which do not allow compensating with a high resolution the measurement errors caused by sporadic ionization of the NEAS. At the same time, one of the possible solutions to this problem is the creation of a network of stations for study of the ionosphere - radio-technical systems that actively influence the ionosphere to determine its parameters to take them into account in systems of location, navigation, television and communication [9].

At the same time, the results provided in $[10,11]$ show that the coordinate data obtained from the ROS can be used in the interests of estimating the parameters of the radio wave propagation medium. For this, it is offered to use a priori coordinate data for those space objects (SO) that are in the ROS coverage regions and whose coordinate data can be measured. By performing the comparison operation, the measurement error introduced by the radio wave propagation medium is calculated, which can then be used in the interests of some radio-technical systems to be considered within their operation. At the same time, implementation of this approach in practice shall consider some aspects, which are briefly discussed below.

\section{INITIAL DATA PREPARATION}

The total electron content (TEC) is known to be a function of the electron concentration of the ionosphere, and its value undergoes significant spatio-temporal fluctuations depending on the heliogeophysical conditions [12]. When comparing coordinate data, it is assumed that the TEC is calculated along the path of 
propagation of the probing and reflected signals. In this case, the position of the trajectory is determined by elevation and azimuth of the SO, from which the reflected signal was received. Therefore, for operation of comparison, it is necessary to obtain data on the position of certain SO.

The best solution is the advance receipt of coordinate data on the ROS, allowing to plan the work on obtaining measurements of the SO position. For this, the data from the catalogs of the $\mathrm{SO}$ or the results of the SO observation by other observation systems can be used $[5,13]$.

A possible solution to this issue is to store an array of data on the radius of the SO orbit, its geodetic latitude and longitude for discrete points in time. In this case, it is offered to use the following expression for preliminary determination of the SO position:

$$
\begin{gathered}
\varepsilon_{i}=\operatorname{arctg}\left(\frac{\cos \Psi_{i}-R_{E} / R_{i}}{\sin \Psi_{i}}\right), \\
\beta_{i}=\arccos \left(\frac{\sin \Phi_{i}-\sin \Phi \cos \Psi_{i}}{\sin \Psi_{i} \cos \Phi}\right), \\
\Psi_{i}=\arccos \left(\sin \Phi \sin \Phi_{i}+\cos \Phi \cos \Phi_{i}\left(\Lambda_{i}-\Lambda\right)\right)
\end{gathered}
$$

Where:

$$
\begin{aligned}
& \Psi_{i} \quad-\text { central angle between the ROS and SO, } \\
& R_{E} \quad-\text { Earth radius, } \\
& R_{i} \quad-\text { orbital radius of the SO, } \\
& \Phi_{i} \quad-\text { geodetic latitude of the SO, } \\
& \Phi \quad-\text { geodetic latitude of the ROS, } \\
& \Lambda_{i} \quad-\text { geodetic longitude of the SO, } \\
& \Lambda \quad-\text { geodetic longitude of the ROS. }
\end{aligned}
$$

The next issue is the TEC value calculation. At the same time, it is offered to use assumption that the integral nature of the TEC does not allow tying the TEC disturbances to the specific range of heights. In [7, 14], it is noted that the largest contribution to the TEC variations falls on the region located near the main maximum of the ionosphere (corresponding to the height $h_{\max }$ ). Therefore, it is offered to use assumption that all TEC variations calculated along the path of propagation of the probing and reflected signals can be related to the height $h_{\max }$. It is assumed that the ionosphere can be considered as a thin layer at a given height, and the propagation trajectory of the probing and reflected ROS signals crosses this layer at the ionospheric point $P$ (Fig. 1). Geographic latitude and longitude of the ionospheric point for the $i$-th SO of $O{ }_{i}$ can be calculated by the expression:

$$
\begin{gathered}
\phi_{i}=\arcsin \left(\sin \phi_{B} \cos \psi_{i}+\cos \phi_{B} \sin \psi_{i} \cos \alpha_{i}\right), \\
\gamma_{i}=\gamma_{B}+\arcsin \left(\sin \psi_{i} \sin \alpha_{i} \sec \phi_{i}\right), \\
\psi_{i}=\frac{\pi}{2}-\varepsilon_{i}-\arcsin \left(\frac{R_{E}}{R_{E}+h_{\max }} \cos \varepsilon_{i}\right) .
\end{gathered}
$$

In this case, preparation of the initial data shall provide for a preliminary assessment of the capabilities of the ROS to ensure a sufficient time resource for servicing the data of the SO. In case of its shortage, it is necessary to rank SO to form such a sample that will remove the uncertainty of the distribution environment in the largest volume of space with the smallest of the possible number of the SO [15].

After preparation of the initial data, they are transmitted for use in radio-technical systems for location, navigation, television and communication for evaluating the parameters of the NEAS and reducing the degree of their negative impact. The issues of using TEC estimates in the interests of various radio-technical systems are considered in detail in [14].

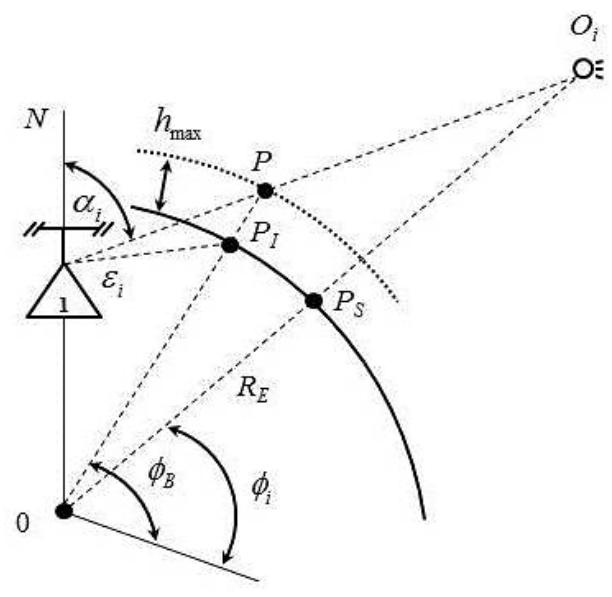

Figure 1 Ionospheric point position calculation

Below we consider one of the options for using the results of the comparison in space-separated radiotechnical systems.

\section{COMPARISON OF COORDINATE DATA WITH SPATIALLY SEPARATED RADIO-TECHNICAL SYSTEMS}

Let's consider a problematic situation using the example of Fig. 2. The ROS located at point $R_{l}$ conducts reconnaissance of the NEAS in a given viewing region. Within the angular dimensions of the ROS coverage region there is navigation satellite $\mathrm{N}_{2}$, according to changes in the signal parameters of which, the measurement errors in a given angular direction are compensated for [14]. In the vicinity of $R_{I}$ there are radio technical systems $S_{1}, S_{2}$ and $S_{3}$, the quality of which depends on the size of the TEC - these can be 
navigation, location, remote sensing, television and communication systems.

According to the ROS of SO $O_{1}$ and $O 2$ located in the viewing region, there is a priori coordinate information which makes it possible to determine the TEC value for points $P_{O 1}$ and $P_{O 2}$, respectively. Movement of the considered SO (as well as others, if any) through the ROS viewing region makes it possible to form an array of data describing the parameters of the ionospheric points at the height $h_{\max }$. This allows, with some spatial and temporal discreteness, to form a region of ionospheric points (RIP), which can be used in the interests of systems $S_{1}, S_{2}$ and $S_{3}$ in the event that the trajectories of the signals of these systems will intersect the RIP. At the same time, $S_{3}$ system is in the most favorable perspective conditions, since the projection of ionospheric points onto the Earth's surface, which makes it possible to form a region of podionospheric points (RPP), in the case of a comparison operation, allows the system to provide information on the TEC values over the entire area.

Determination of the TEC value at the required point can be carried out by interpolation methods (Fig. 3) using expression 2 .

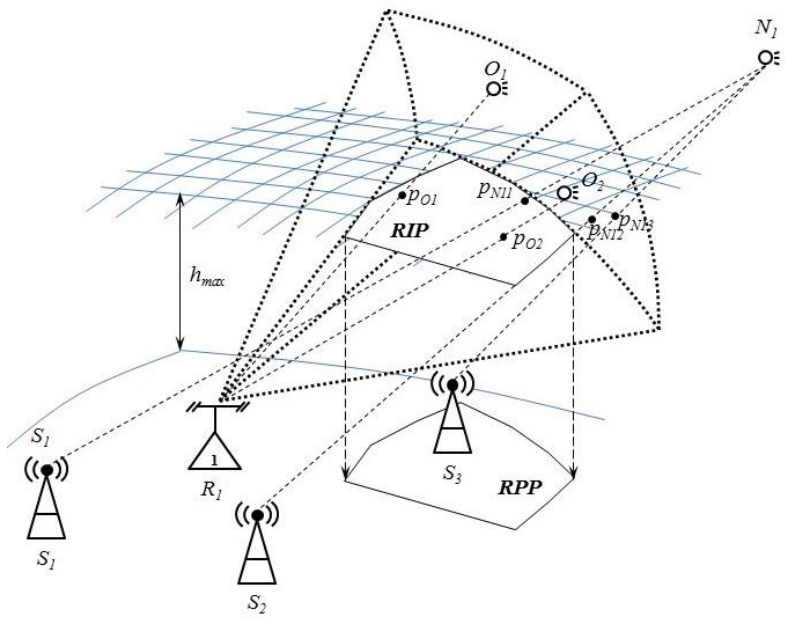

Figure 2 Comparison of coordinate data with spatially separated radio-technical systems

Preliminary calculations have shown [15] that the comparison operation can give an increase of up to 12$18 \%$ to the volume of TEC estimates, including in critical regions of lack of data that do not cross the radio lines "navigation satellite - signal receiver" [16]. In this case, it is only necessary to refine the ROS software using the example of the algorithmic implementation of coordinate data comparison considered in [17], as well as to create information exchange channels with nearby radio-technical systems for the prompt provision of the comparison results.

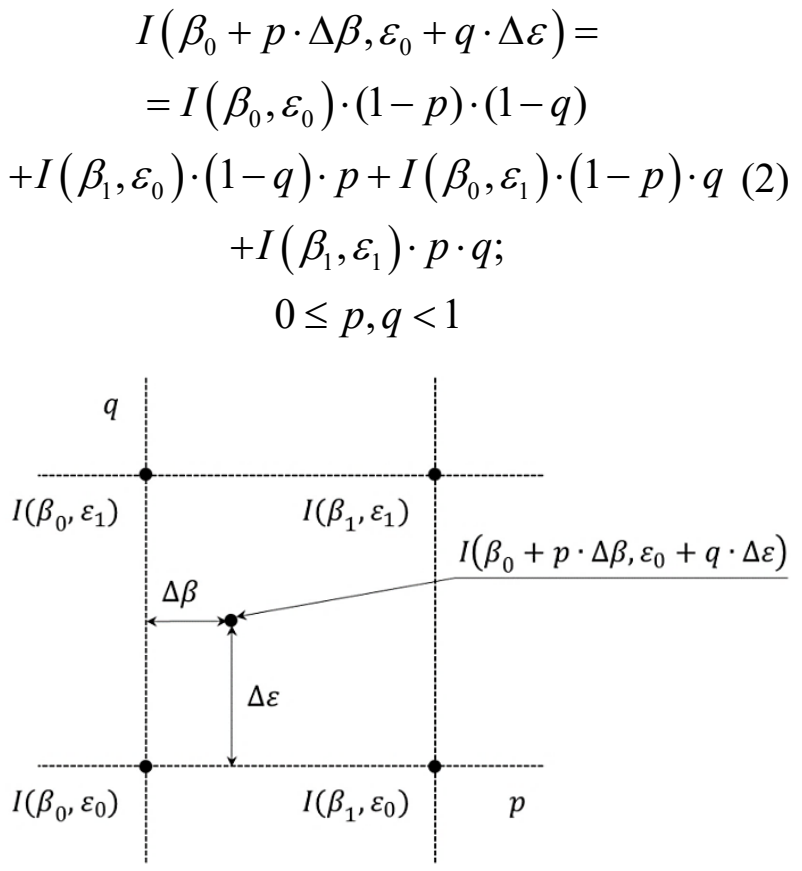

Figure 3 Calculation of TEC values at the interpolation point

In addition, a promising direction in considering the influence of the propagation medium is use of new approaches to processing of radar information [18] to identify systematics in measurement errors to take it into account when processing measurement results from the corresponding regions of space.

\section{CONCLUSIONS}

Within the framework of the article, some aspects of the coordinate data comparison by radio-technical systems are considered. The features of preparation of the initial data and the structure of spatially separated radio-technical systems are described, which allow to determine the TEC in a given angular direction from the error in measuring the SO position which was introduced by the propagation medium. It is shown that the calculated TEC values can be used in the interests of location, navigation, remote sensing, television and communications systems to take into account the negative impact of the propagation environment during their operation. This, in turn, makes it possible to reduce the need for creation of new ROS by increasing the detection accuracy of existing ones, as well as to increase the levels of safety and favorable conditions for human life within implementation of urban planning activities by improving the quality of functioning of radio-technical systems for location, navigation, television and communications.

\section{REFERENCES}

[1] Urban Planning Code of the Russian Federation dated December 29, 2004 No. 190-FZ (2012) 
[2] V.V. Adushkin, O.Yu. Aksenov, S.S. Veniaminov, S.I. Kozlov, On danger of small space debris for space activities and the ecology of the Earth. In: Aerospace sphere, 3(100) (2019) pp. 73-81.

[3] V.V. Adushkin, O.Yu. Aksenov, S.S. Veniaminov, S.I. Kozlov, On approaches to assessing the potential danger of destruction and collisions of space objects. In: Vozdushno-kosmicheskaya sphera, 1(94) (2018) pp. 54-63.

[4] A. V. Denisov, V.V. Popov, S.V. Logunov, P.V. Karev, Optical-electronic complex of detailed surveillance. Scientific and Technical Journal of Information Technologies, Mechanics and Optics, 20(1) (2020) pp.24-31. DOI: $10.17586 / 2226-$ 1494-2020-20-1-24-31

[5] V.M. Agapov, A.V. Golovko, V.A. Emelyanov, V.P. Konoshenko, A.A. Kuriksha, S.S. Loginov, Yu.N. Makarov, Yu.K. Merkushev, M.A. Mikhailov, I.E. Molotov, Space Debris. In 2 books. Book 1. Methods of observation and models of space debris: monograph, 248, 2014.

[6] A.F. Avtushenko. Powerful over-the-horizon early warning radars: development, testing, operation: monograph, Radiotekhnika Publishing house, 2013, p. 168.

[7] A. Devyatkin, I. Gotur, V. Krasnov, Y. Kuleshov, A. Meshkov Ionospheric total electron content modelling and the estimation of errors in coordinate measuring caused by solar activity in remote sensing, International journal of remote sensing 35(15) (2014) pp. 5913-5925. ISSN: 01431161

[8] E.L. Afraimovich, V.A. Karachentsev, Errors in the functioning of satellite radio engineering systems due to the state of the transionospheric channel. In: Electronic Magazine Investigated in Russia, 6 (2003)

pp. 2382-2390. http://zhurnal.ape.relarn.ru/articles/2003/202.pdf.

[9] V.P. Lebedev, A.V. Medvedev, M.V. Tolstikov, Interference measurements of ionospheric disturbances on the Irkutsk incoherent scattering radar. In: Bulletin of the Siberian State University named after M.F. Reshetnev, 5(51) (2013) pp. 166169.

[10] N.A. Kupriyanov, R.S. Misko, Approach to use of cataloged space objects in the interests of consumers of navigation information. In: Proceedings of the 75th Scientific and Technical Conference of the Scientific and Technical Society of Radio Engineering, Electronics and Communications named after A.S. Popov (St. SPB NTORES) dedicated to Radio Day, pp. 36-38, 2020.
[11] Yu.V. Zheleznyakov, N.A. Kupriyanov, E.V. Okunev, Approach to calculating the trajectory of an object by a tracking radar system using a priori coordinate data. In: Questions of control of economic activities and financial audit, national security, system analysis and management: Proceedings of materials of the $\mathrm{V}$ All-Russian Scientific-Practical Conference; Moscow, December 27 (2019) pp. 283-286.

[12] V.V. Demyanov, I.K. Edemsky, A.A. Markidonova, N.P. Perevalova, Yu.V. Yasyukevich, Investigation of near-Earth space using global satellite navigation systems. In: Bulletin of the Siberian State University named after M.F. Reshetnev, 6(52) (2013) pp. 94-100.

[13] M.E. Prokhorov， A.I. Zakharov， A.V. Mironov, A.V. Biryukov, O.Yu. Stekol'shchikov, M.S. Tuchin, A.O. Zhukov, The possibility of a deep scanning survey of part of the sky from a loworbit spacecraft with fixed orbital orientation. In: Baltic Astronomy, 24 (2015) p. 92-99.

[14] A.M. Vesnin, I.K. Edemsky, I.V. Zhivetyev, D.S. Kotova, A.A. Mylnikova, V.B. Ovodenko, Yu.V. Yasyukevich, Methods of compensation for the ionospheric component of the error of radio engineering systems using data of full electronic content GPS / GLONASS. In: Bulletin of the Volga State Technological University. Ser.: Radio engineering and information systems, 2(34) (2017) pp. 19-31.

[15] N.A. Kupriyanov, S.V. Logunov, Methods for ranking cataloged space objects used to improve the accuracy of determining the coordinates of objects by an early warning radar station. In: Issues of radio electronics, ser. Television equipment, 1 (2019) pp. 75-85.

[16] V.E. Kunitsyn, I.A. Nesterova, E.S. Andreeva, Radiotomographic studies of the ionosphere according to navigation satellite systems. In: AllRussian Scientific Zvorykin Readings - VI, pp. 1219, 2014.

[17] D.N. Vorobiev, N.A. Kupriyanov, A.Yu. Onufrey, Algorithmic implementation of comparison of trajectory data by an early warning radar station. In: Issues of radio electronics, ser. Television equipment, 4 (2020) pp. 93-100.

[18] N.S. Kondybaev， N.A. Kupriyanov， S.Z. Kurakin, Algorithm for trajectory processing of information from radar measuring complexes based on clustering by the K-MEANS method. In: Scienceintensive technologies in space research of the Earth, 12(6) (2020) pp. 4-10. 